\title{
Wake Measurements behind an Oscillating Airfoil in Dynamic Stall Conditions
}

\author{
A. Zanotti, G. Gibertini, D. Grassi, and D. Spreafico \\ Politecnico di Milano, Dipartimento di Scienze e Tecnologie Aerospaziali, Campus Bovisa, Via La Masa 34, 20156 Milano, Italy \\ Correspondence should be addressed to A. Zanotti; alex.zanotti@polimi.it
}

Received 4 March 2013; Accepted 3 April 2013

Academic Editors: J. Meseguer, R. K. Sharma, and W. Zhang

Copyright ( 2013 A. Zanotti et al. This is an open access article distributed under the Creative Commons Attribution License, which permits unrestricted use, distribution, and reproduction in any medium, provided the original work is properly cited.

\begin{abstract}
The unsteady flow field in the wake of an NACA 23012 pitching airfoil was investigated by means of triple hot-wire probe measurements. Wind tunnel tests were carried out both in the light and deep dynamic stall regimes. The analysis of the wake velocity fields was supported by the measurements of unsteady flow fields and airloads. In particular, particle image velocimetry surveys were carried out on the airfoil upper surface, while the lift and pitching moments were evaluated integrating surface pressure measurements. In the light dynamic stall condition, the wake velocity profiles show a similar behaviour in upstroke and in downstroke motions as, in this condition, the flow on the airfoil upper surface is attached for almost the whole pitching cycle and the airloads show a small amount of hysteresis. The deep dynamic stall measurements in downstroke show a large extent of the wake and a high value of the turbulent kinetic energy due to the passage of strong vortical structures, typical of this dynamic stall regime. The comprehensive experimental database can be considered a reference for the development and validation of numerical tools for such peculiar flow conditions.
\end{abstract}

\section{Introduction}

The aerodynamics of oscillating airfoils is widely investigated as it represents a good model for the study of the dynamic stall of the retreating blade sections $[1,2]$. The dynamic stall phenomenon occurs on the retreating side of the helicopter rotor at a high forward flight speed or during maneuvers at high load factors, and it produces several adverse effects on the helicopter performance. The main detrimental effects due to dynamic stall are the limitation of the forward speed and thrust, high control system loads, the introduction of a high level of vibrations affecting the helicopter dynamic performance in terms of maneuver capability and handling qualities, and the occurrence of the aeroelastic problem known as stall flutter [3] causing blade structural damage and excessive cabin vibration.

Therefore, in order to overcome the limitations on helicopter performance introduced by this phenomenon and to expand the helicopter flight envelope and vehicle utility, dynamic stall has become in the recent years one of the most challenging research topics in rotorcraft aerodynamics field. In fact, several research activities both in experimental and numerical fields investigated the effectiveness of different dynamic stall control systems integrated into the blade section (e.g., [4-6]). Moreover, the study of the fine details involved in the physics of this phenomenon was widely investigated in the recent years, as, for instance, in [7-9]. In this research area, the study of the unsteady wake of an oscillating airfoil features a relatively low attention by the literature with respect to the evaluation of the characteristic flow field and the measurement of the airloads on oscillating airfoils in dynamic stall conditions, as demonstrated by the large number of research activities focused on this topic (e.g., [9-12]).

In the recent literature, Jung and Park [13] investigated the unsteady characteristics of the vortex shedding in the near wake of an oscillating airfoil by means of hot-wire anemometry surveys and smoke visualizations, finding that the vortex-shedding characteristics in dynamic conditions are quite different from the steady airfoil case and depends on the state of the boundary layer. Hot-wire measurements were also carried out by Chang [14] to study the Reynolds number effects and the turbulence intensity in the near wake of a NACA 4412 oscillating airfoil. Moreover, Sadeghi et al. [15] 
investigated the effects of the operating conditions of the pitching airfoil, as mean angle of attack, reduced frequency, and instantaneous angle of attack on the wake velocity field. In a more recent work by Sadeghi et al. [16], wake measurements behind an oscillating airfoil compared to smoke visualizations were considered to predict the aerodynamic features characteristic of the dynamic stall phenomenon.

The present paper presents the results of an experimental activity that investigated the flow conditions evolution around an NACA 23012 airfoil during its pitching oscillation. The wind tunnel tests were carried out at the Aerodynamics Laboratory of Politecnico di Milano, using an experimental rig designed for testing full-scale helicopter blade sections oscillating in the pitch. In particular, the wake of the pitching blade section model was measured by means of a triple hot-wire probe. The test campaign investigated the different regimes of the dynamic stall occurring on the rotor retreating blade in a forward flight, namely, the light and deep dynamic stall [1]. The analysis of the wake velocity fields was supported by the measurements of unsteady flow fields and airloads [17]. In particular, the flow fields over the upper surface of the oscillating airfoil were measured by means of particle image velocimetry (PIV). Lift and pitching moments were evaluated integrating surface pressure measurements carried out on the midspan contour of the model by means of fast unsteady pressure transducers. The experimental investigation was completed by unsteady pressure measurements carried out on the wind tunnel floor and ceiling.

\section{Experimental Setup}

The experimental activity was conducted at Politecnico di Milano in the low-speed closed-return wind tunnel of the Aerodynamics Laboratory of DAER. The wind tunnel has a rectangular test section with $1.5 \mathrm{~m}$ height and $1 \mathrm{~m}$ width. The maximum wind velocity is $55 \mathrm{~m} / \mathrm{s}$, and the freestream turbulence level is less than $0.1 \%$.

An NACA 23012 aluminium machined model, with chord $c=0.3 \mathrm{~m}$ and $\operatorname{span} b=0.93 \mathrm{~m}$, was used in this activity. The airfoil model has an interchangeable midspan section for the different measurements' techniques employed, one for PIV flow surveys and another for unsteady pressure measurements equipped with pressure taps positioned along the midspan contour. At the maximum angle of the attack tested $\left(\alpha=20^{\circ}\right)$, the wind tunnel blockage due to the oscillating airfoil model is $6 \%$. The model is pivoted around two external steel shafts with the axis at $25 \%$ of the airfoil chord. The pitching drive system is composed of a brushless servomotor with a 12:1 gear drive jointed mechanically to one of the model external steel shafts. The end plates were mounted at model extremity to reduce the interference of the wind tunnel walls' boundary layer and to reduce the extremity effects. Two encoders are directly mounted on the shaft on the other side of the model respective to the motor: a $2048 \mathrm{imp} / \mathrm{rev}$ absolute digital encoder with EnDat 2.2 protocol is used for feedback control, and a $4096 \mathrm{imp} / \mathrm{rev}$ incremental analog encoder is used to get the instantaneous position of the model. Further details about the pitching airfoil experimental rig can be found in $[18,19]$. The layout of the experimental rig designed for testing pitching airfoils is illustrated in Figure 1.

2.1. Hot-Wire Measurement Setup. The velocity surveys in the wake of the oscillating airfoil were carried out by means of a constant temperature anemometry (CTA) system Streamline 90 N10 by Dantec Dynamics. The system was composed of a one frame with three CTA modules. Every basic anemometer module contains three CTA bridges, a servo loop with programmable gain, filters and cable compensation, and a programmable signal conditioner. The programmable servo loop allows to optimize the dynamic response and the bandwidth of the system, while the signal conditioner provides amplification of the CTA signal before digitizing.

A tri-axial fiber-film probe, Dantec 55R91, was used for the velocity surveys. The sensor probe has three mutually perpendicular sensors, consisting of $70 \mu \mathrm{m}$ diameter nickelplated quartz fibres. The probe was calibrated in the laboratory under monitored conditions with respect to Reynolds number and velocity direction. The calibration method took into account the effects of temperature, pressure, and humidity in order to extend the calibration itself to the wind tunnel ambient conditions [20]. The probe was mounted on a supporting strut, and it was moved in the model midspan plane in a vertical direction by means of a single axis traversing system. The velocity profiles were measured 2 chords past the airfoil trailing edge. The velocity time history was acquired for a time corresponding to 150 complete pitching cycles with a sampling frequency of $20 \mathrm{kHz}$.

Figure 2 illustrates the blade section model inside the wind tunnel; behind the model, the supporting strut for the triple hot-wire probe can be observed.

2.2. PIV Setup. The PIV system used a double shutter CCD camera with a 12-bit, $1280 \times 1024$-pixel array and a $55 \mathrm{~mm}$ lens. The measurement area covers the airfoil upper surface and it was composed by different measurement windows spanning the chord direction to obtain image pairs with a better resolution. The CCD camera was mounted on a dual axis traversing system driven by two stepper motors that allowed to move the measurement window along two orthogonal guides.

An Nd:Yag double pulsed laser, with $200 \mathrm{~mJ}$ output energy and wavelength $532 \mathrm{~nm}$, was positioned on the test section roof. The laser was mounted on a single axis traversing system to move the sheet along the wind tunnel flow direction. This solution enabled to use a laser sheet with a smaller width and a higher energy level centered on each measurement window. A particle generator with Laskin nozzles was used for the flow insemination. The tracer particles, consisting of small oil droplets with a diameter in the range of $1-2 \mu \mathrm{m}$, were injected in correspondence to a section just after the fans and fulfill the wind tunnel volume with a homogeneous density.

The velocity flow fields phase which averaged over 40 image pairs are presented in the following. 


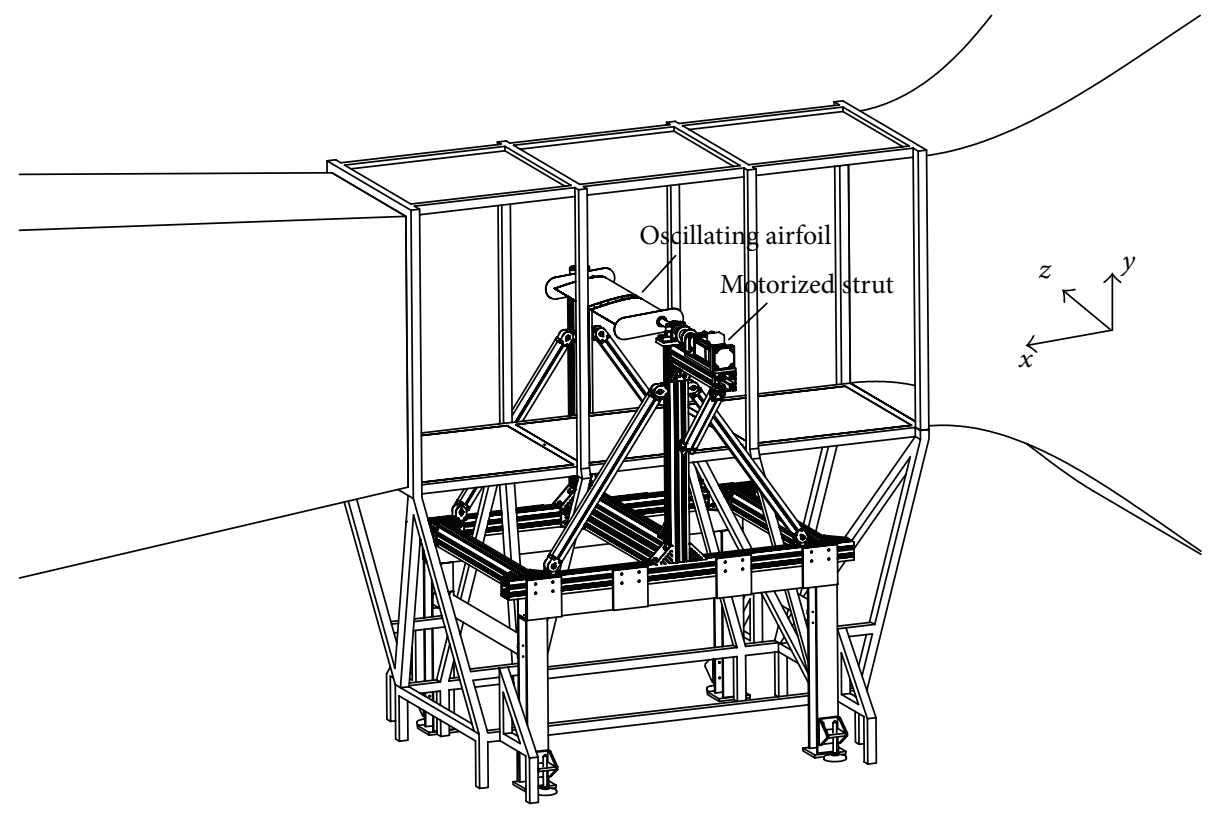

FIGURE 1: Layout of the experimental rig for pitching airfoils tests.

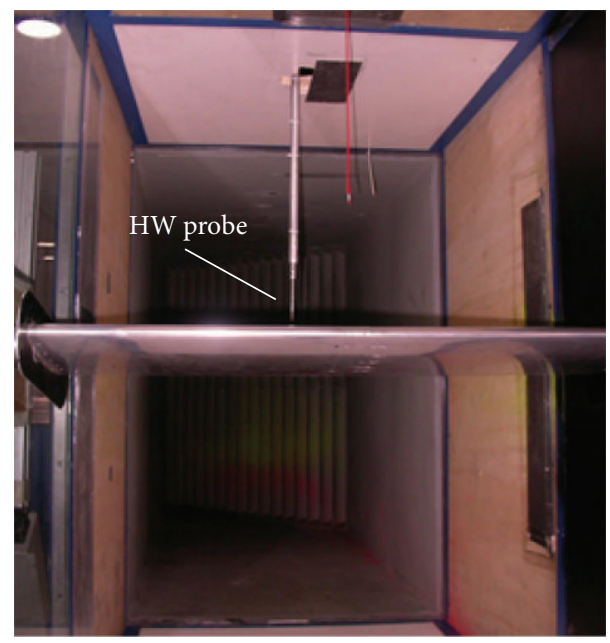

FIGURE 2: NACA 23012 blade section model inside the wind tunnel test section.

\subsection{Pressure Measurement Setup}

2.3.1. Airfoil Model Surface. The time history of the lift and pitching moments during a pitching cycle was evaluated by the integration of the phase-averaged pressures collected over 30 complete pitching cycles. Pressure was measured on the airfoil midspan contour using 21 Kulite fast-response pressure transducers located inside the model interchangeable midspan section. The locations of the pressure taps around the model midspan section are listed in Table 1 following a closed loop from the upper to the lower surface of the airfoil, starting from the leading edge.

The pressure transducers signals were acquired with a sampling rate of $50 \mathrm{kHz}$ by means of a National Instruments compact data acquisition system equipped with six 24-bit A/D simultaneous bridge modules with 4 channels each.

2.3.2. Wind Tunnel Ceiling and Floor. Pressure measurements were carried out also on the wind tunnel ceiling and floor by means of a pressure rod (see Figure 3(a)). The rod was instrumented with two Kulite fast-response transducers ( 2 psi F.S.), and it was mounted on the test section ceiling and the floor in two different tests for each investigated dynamic stall condition. The pressure taps were located on the longitudinal midspan plane of the rod, while the transducers were installed in a threaded housing on the lateral side of the rod as it can be observed from the particular of the layout in Figure 3(b). Pressure was measured in correspondence to two pressure taps located 2 chords downstream the airfoil trailing edge (longitudinal position of the HW velocity surveys) and 3 chords upstream the airfoil leading edge.

\section{Phase Averaging Method}

The phase averaging is the most widely used method to point out a time-varying signal measured in the case of periodic unsteady flows. The measured time-varying signal $s(t)$ can be decomposed as follows:

$$
s(t)=\langle s(t)\rangle+s^{\prime}(t)
$$

into a phase average term $\langle s(t)\rangle$ and a fluctuating term $s^{\prime}(t)$. The phase averaging operator is defined as follows:

$$
\langle s(t)\rangle=\lim _{N \rightarrow \infty} \frac{1}{N} \sum_{i=1}^{N} s(t+(i-1) T),
$$


TABLE 1: Pressure taps location on the NACA 23012 model midspan section.

\begin{tabular}{lc}
\hline Tap number & Location $x / c$ \\
\hline 1 & 0 \\
2 & 0.01 \\
3 & 0.044 \\
4 & 0.096 \\
5 & 0.164 \\
6 & 0.28 \\
7 & 0.358 \\
8 & 0.453 \\
9 & 0.618 \\
10 & 0.76 \\
11 & 0.9 \\
12 & 0.9 \\
13 & 0.767 \\
14 & 0.628 \\
15 & 0.459 \\
16 & 0.373 \\
17 & 0.285 \\
18 & 0.185 \\
19 & 0.118 \\
21 & 0.06 \\
\hline
\end{tabular}

where $T$ is the period of the cyclic flow and $N$ is the number of cycles, while the fluctuations term is defined as

$$
s^{\prime}(t)=s(t)-\langle s(t)\rangle .
$$

In practice, the phase average term depends also on the number of cycles $N$ as the phase averaging method is carried out over a finite number of cycles. Then, the definition of the phase average term in (2) becomes

$$
\langle s(t, N)\rangle=\frac{1}{N} \sum_{i=1}^{N} s(t+(i-1) T) .
$$

The larger is the number of cycles $N$, the more converging is the $\langle s(t, N)\rangle$ value towards the theoretical phase average term $\langle s(t)\rangle$. The criterion to determine the number of cycles to be used in the phase averaging method was discussed by Wernert and Favier [21] for different measurement techniques.

\section{Experimental Results}

The two conditions considered for wake velocity surveys are sinusoidal pitching cycles with reduced frequency $k=0.1$, oscillation amplitude $\alpha_{a}=10^{\circ}$, and a mean angle of attack of $\alpha_{m}=5^{\circ}$ and $\alpha_{m}=10^{\circ}$ to reproduce, respectively, the light and the deep dynamic stall regimes. The tests were carried out at $U_{\infty}=30 \mathrm{~m} / \mathrm{s}$ corresponding to a Reynolds number Re = $6 \times 10^{5}$ and a Mach number Ma $=0.09$. Such a low testing speed, which is far beyond the test rig capabilities, was chosen because the long run time required by the HW wake surveys might have raised fatigue issues on the model strut.

Figures 4 and 5 present the phase averaged freestream velocity component profiles measured in the wake of the oscillating airfoil at some selected angles of attack. The wake freestream velocity component was phase averaged over 150 pitching cycles.

For the test condition with $\alpha_{m}=5^{\circ}$, the measured velocity profiles show a small defect of the freestream velocity component, both in upstroke and in downstroke (see Figure 4). The similar behavior shown by the velocity profiles with respect to the direction of the airfoil oscillating motion is in agreement with the behavior of the measured airloads. In fact, as it can be observed in Figures 6(a) and 6(b), for this light dynamic stall condition, both the measured $C_{L}-\alpha$ and $C_{M}-\alpha$ curves show a small amount of hysteresis.

Moreover, the vertical extent of the wake velocity defect region is in the order of the airfoil thickness, both in upstroke and in downstroke. Indeed, in this dynamic stall regime the flow on the airfoil upper surface is attached for almost all the pitching cycle, as it can be observed by PIV surveys carried out on the model midspan plane. The PIV results in the following are illustrated by means of the in-plane streamlines patterns. For this light dynamic stall condition, the flow over the upper surface of the airfoil is fully attached at $\alpha=10^{\circ}$ both in upstroke and downstroke (see Figures $7(\mathrm{a})$ and $7(\mathrm{c})$ )), as well as at the top of the upstroke motion $\alpha=15^{\circ}$ (see Figure 7(b)).

For the test condition with $\alpha_{m}=10^{\circ}$, the velocity profiles behavior in the upstroke motion is similar to the one measured for the light dynamic stall condition (see Figure 5). The wake profiles measured in upstroke are coherent with the predominant aerodynamic feature due to the dynamic stall phenomenon. Indeed, the stall is delayed with respect to the static case due to a kinematic-induced camber increase produced by the rapid positive pitching rate [2]. As it can be observed from Figure 6(a), the lift grows linearly to a maximum value higher than the static stall value. In this test condition, the flow is attached to the airfoil upper surface for almost all the upstroke motion, as it is illustrated by the PIV survey carried out at $\alpha=18^{\circ}$ in upstroke (see Figure 8(a)).

During the downstroke motion, the measured velocity profiles show a higher velocity defect and a higher thickness of the wake with respect to the upstroke. The wake thickness can be considered a parameter to estimate the size of the vortical flow in the wake [13]. Indeed, in this phase of the motion, the flow field on the airfoil upper surface shows a large separation, and it is characterised by the rapid formation, migration, and shedding of strong vortices. These flow phenomena are typical of the deep dynamic stall regime [2], and they are responsible of the conspicuous and rapid variations of the airloads measured during this phase of the pitching motion (see Figures 6(a) and 6(b)). Moreover, the observed asymmetric behavior of the flow field with respect to the direction of the airfoil motion results in the large airloads' hysteresis observed in Figures 6(a) and 6(b).

In particular, at $\alpha=15^{\circ}$, the wake thickness reaches about half of the test section height, as it can be observed 


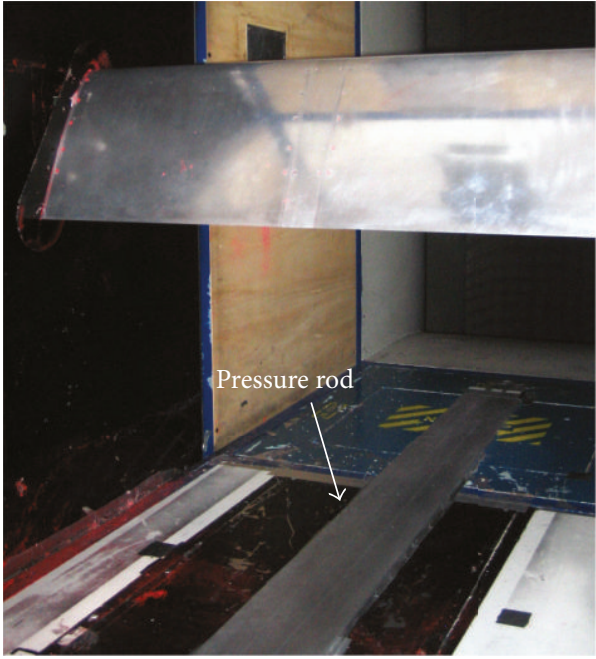

(a)

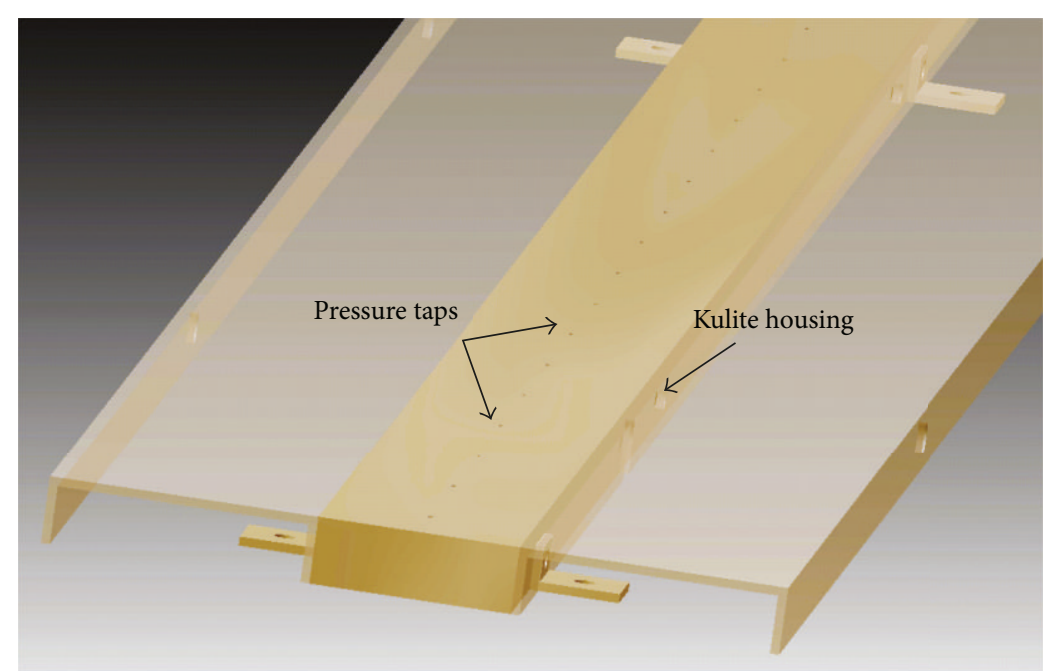

(b)

FIGURE 3: (a) Aluminium rod for pressure measurements installed in the test section; (b) particular of the pressure rod.

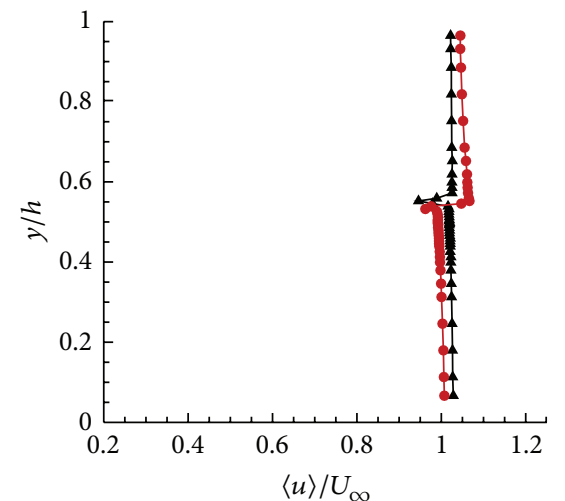

(a) $\alpha=-4^{\circ}$

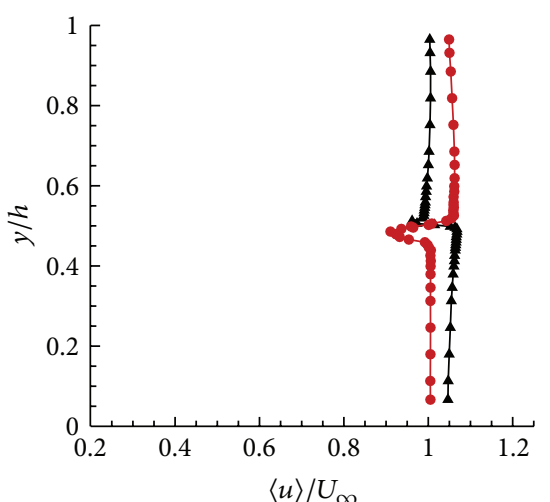

$\langle u\rangle / U_{\infty}$

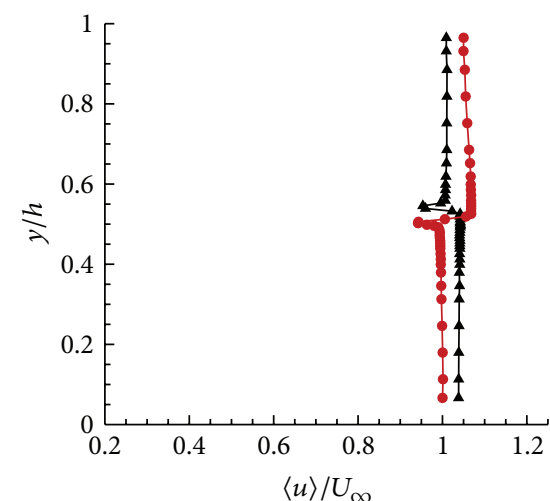

(b) $\alpha=0^{\circ}$

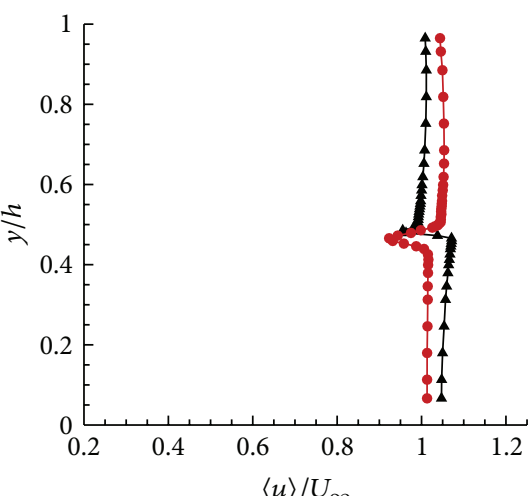

$\langle u\rangle / U_{\infty}$

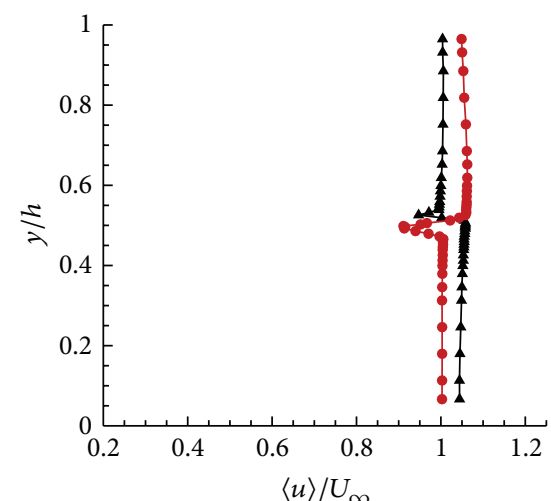

(c) $\alpha=4^{\circ}$

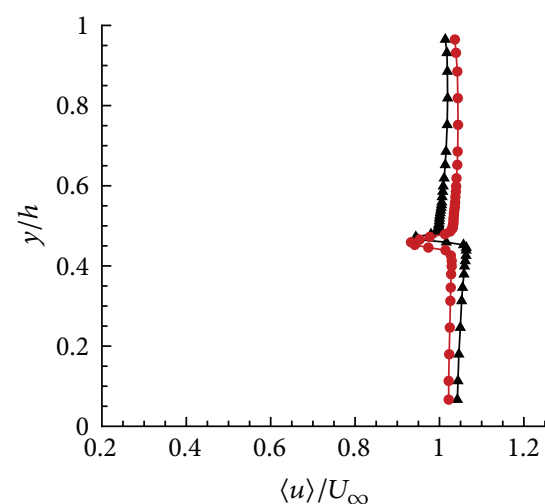

$\neg$ Upstroke

- Downstroke

(f) $\alpha=14^{\circ}$

(d) $\alpha=8^{\circ}$

(e) $\alpha=12^{\circ}$

FIGURE 4: Streamwise velocity profiles for $\alpha=5^{\circ}+10^{\circ} \sin (\omega t)$ and $k=0.1\left(\mathrm{Re}=6 \times 10^{5}\right.$ and $\left.\mathrm{Ma}=0.09\right)$. 


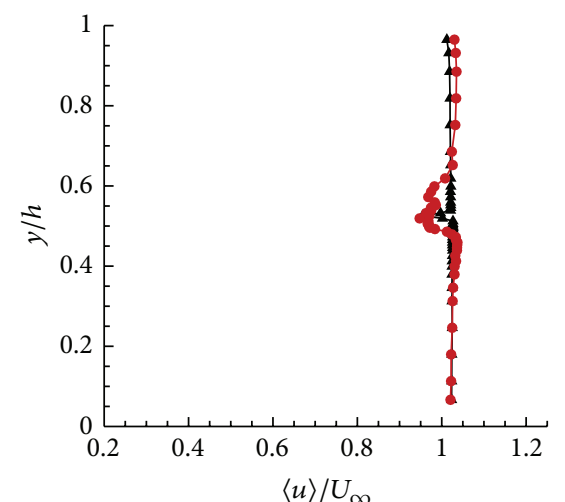

(a) $\alpha=2^{\circ}$

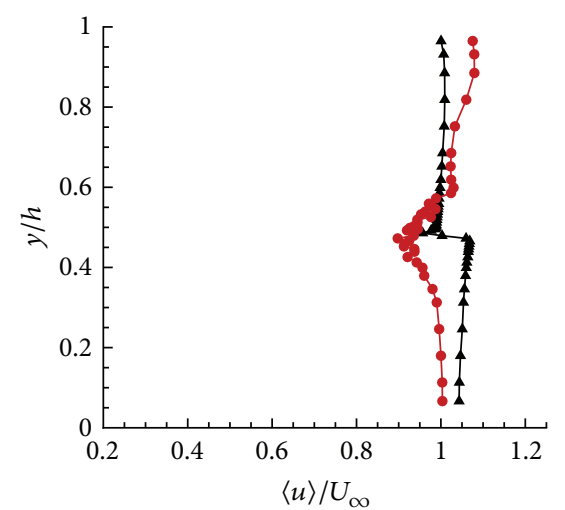

$\langle u\rangle / U_{\infty}$

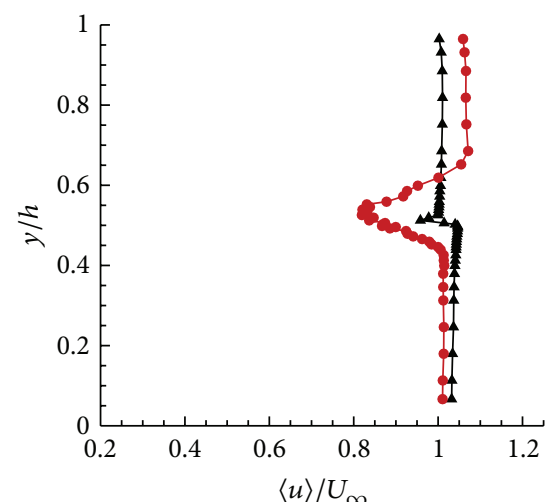

(b) $\alpha=6^{\circ}$

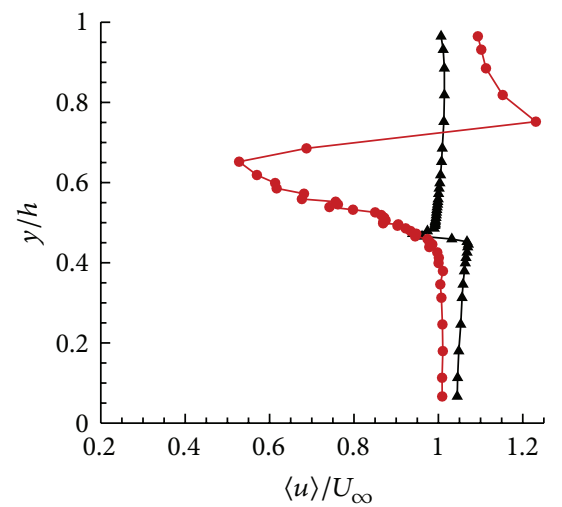

$\langle u\rangle / U_{\infty}$

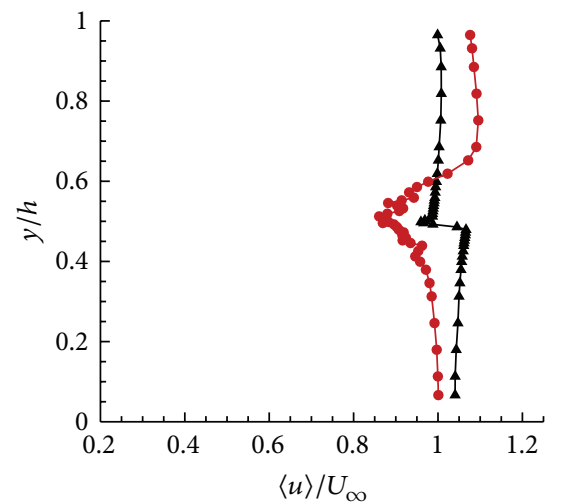

(c) $\alpha=10^{\circ}$

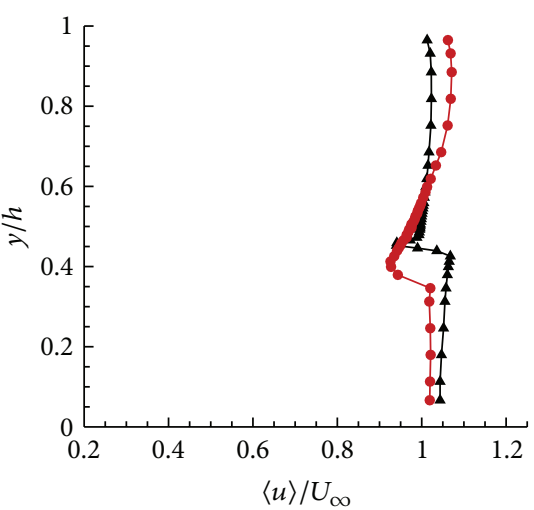

$\_$Upstroke

$\rightarrow$ Downstroke

(f) $\alpha=18^{\circ}$

(d) $\alpha=12^{\circ}$

(e) $\alpha=15^{\circ}$

FIGURE 5: Streamwise velocity profiles for $\alpha=10^{\circ}+10^{\circ} \sin (\omega t)$ and $k=0.1\left(\operatorname{Re}=6 \times 10^{5}\right.$ and $\left.\mathrm{Ma}=0.09\right)$.

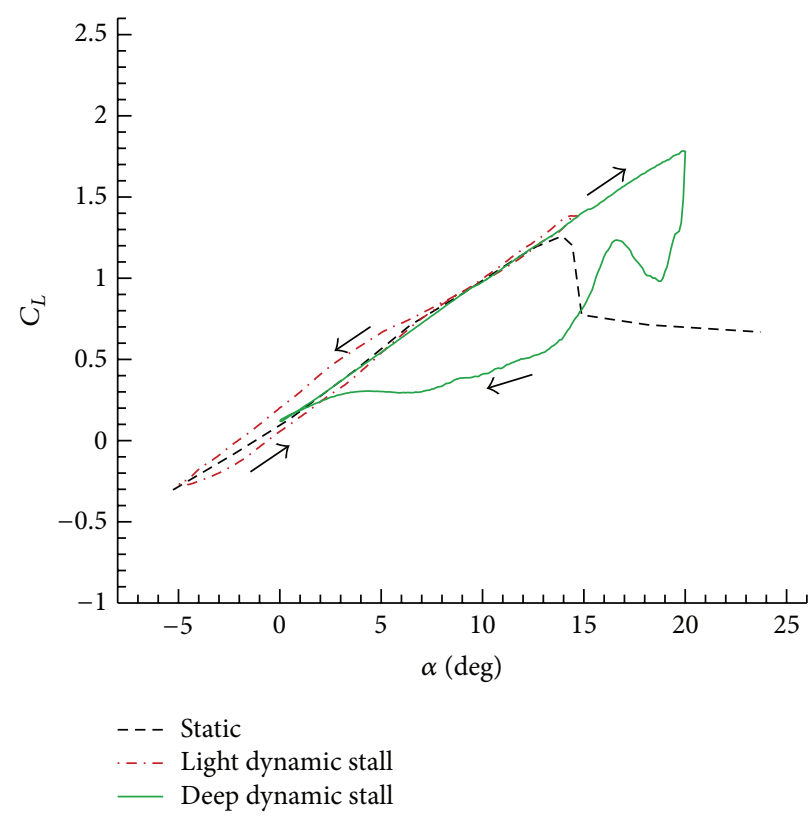

(a)

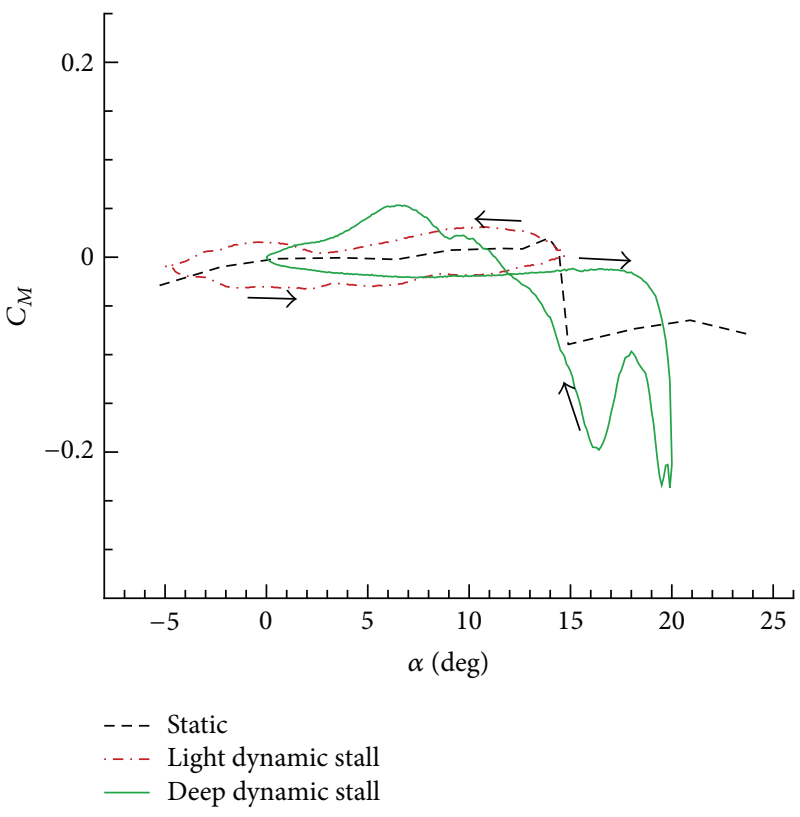

(b)

Figure 6: $C_{L}-\alpha$ and $C_{M}-\alpha$ curves for the NACA 23012 airfoil in static and dynamic stall conditions $\left(\operatorname{Re}=6 \times 10^{5}\right.$ and Ma $\left.=0.09\right)$. 


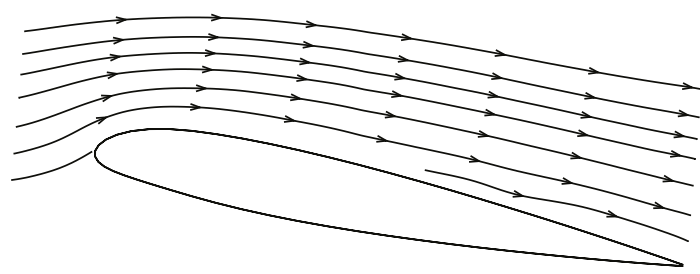

(a) $\alpha=10^{\circ}$ upstroke

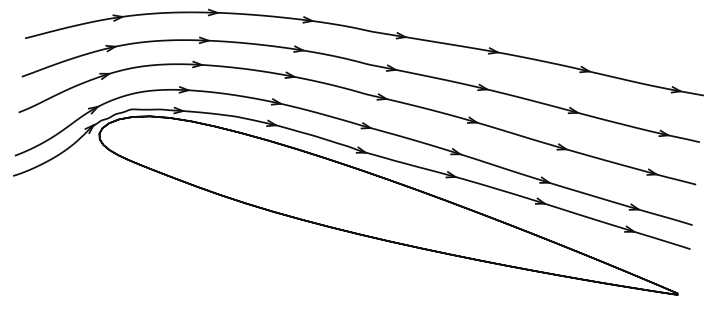

(b) $\alpha=15^{\circ}$

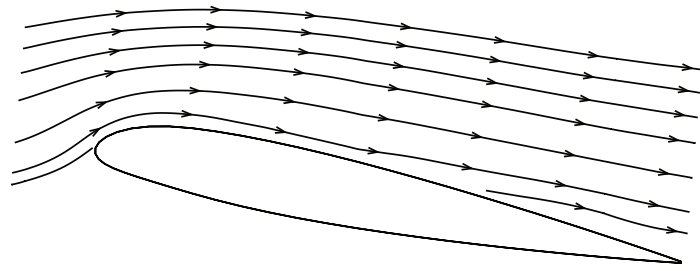

(c) $\alpha=10^{\circ}$ downstroke

Figure 7: PIV results for $\alpha=5^{\circ}+10^{\circ} \sin (\omega t)$ and $k=0.1(\operatorname{Re}=$ $6 \times 10^{5}$ and $\mathrm{Ma}=0.09$ ).

in Figure 5(e). The huge thickness of the wake evaluated at this angle of the attack is due to the passage of a strong vortical structure that starts on the airfoil upper surface at the beginning of the downstroke, as it can be observed from the PIV survey carried out at $\alpha=18^{\circ}$ and $\alpha=16^{\circ}$ in downstroke. At the lower angles of the attack in downstroke (see Figures 5(a) and 5(b)), the wake thickness tends to decrease according to the flow reattachment process start.

It is apparent from Figures 4 and 5 that the velocity profiles settle at two different values towards the wind tunnel floor and ceiling. A similar pattern is commonly observed in wind tunnel tests due to the flow circulation around the model interacting with the tunnel walls (see, e.g., [22]). In the present case, this up-down velocity difference is mainly due to the circulation in the wake [23]. In fact, for the case of a pitching airfoil with a high oscillation amplitude, a rapid circulation variation on the airfoil is associated with the lift variation, and consequently an opposite circulation is issued in the wake. Thus; in the upstroke phase, when the lift is increasing, a counterclockwise circulation is issued in the wake producing higher velocities on the floor with respect to the ceiling and vice versa in the downstroke phase.

This effect can also explain most of the difference between the ceiling and floor pressures highlighted by the pressure measurements results shown in Figure 9. In particular, Figure 9 presents the difference of the phase averaged pressures measured upstream and downstream the airfoil both on

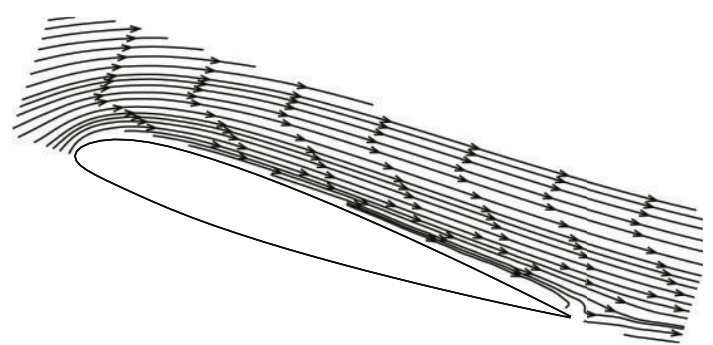

(a) $\alpha=18^{\circ}$ upstroke

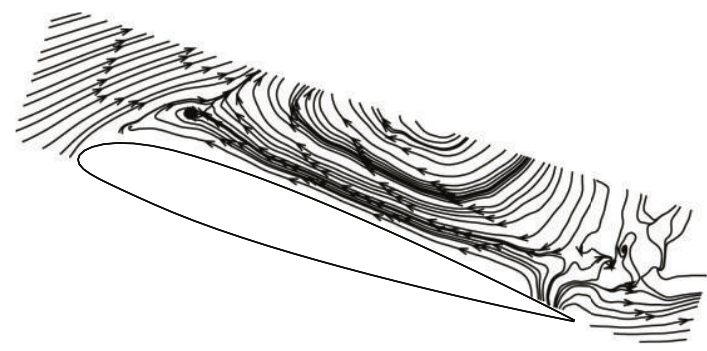

(b) $\alpha=18^{\circ}$ downstroke

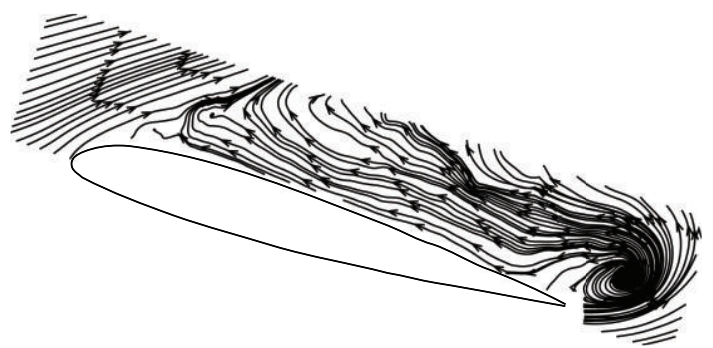

(c) $\alpha=16^{\circ}$ downstroke

Figure 8: PIV results for $\alpha=10^{\circ}+10^{\circ} \sin (\omega t)$ and $k=0.1(\operatorname{Re}=$ $6 \times 10^{5}$ and $\mathrm{Ma}=0.09$ ).

the ceiling and the floor of the test section for the different investigated dynamic stall conditions.

Further considerations about the pitching airfoil wakes in light and deep dynamic stall conditions can be deduced from the analysis of Figures 10 and 11, showing, respectively, the evolution of the adimensional freestream velocity defect $\widehat{U}_{\text {def }}$ and of the adimensional turbulent kinetic energy $\widehat{u}^{\prime}$ during the pitching cycle, where

$$
U_{\mathrm{def}}=1-\frac{\langle U\rangle}{U_{\infty}}, \quad e_{k}=\frac{1}{2} \frac{\left\langle u^{\prime} u^{\prime}\right\rangle\left\langle v^{\prime} v^{\prime}\right\rangle}{U_{\infty}^{2}} .
$$

For the light dynamic stall condition, the defect velocity region moves along the vertical direction of the test section showing the wake oscillations. The more extended region of the velocity defect observed during the downstroke demonstrates a small thickening of the wake in this phase of the motion (see Figure 10(a)). In this test case, the level of the turbulent kinetic energy is quite low for the whole pitching cycle (see Figure 11(a)).

For the deep dynamic stall condition, the velocity defect evolution presents a similar behavior with respect to the light dynamic stall case. On the other hand in downstroke, the velocity defect behavior shows the conspicuous thickening of 


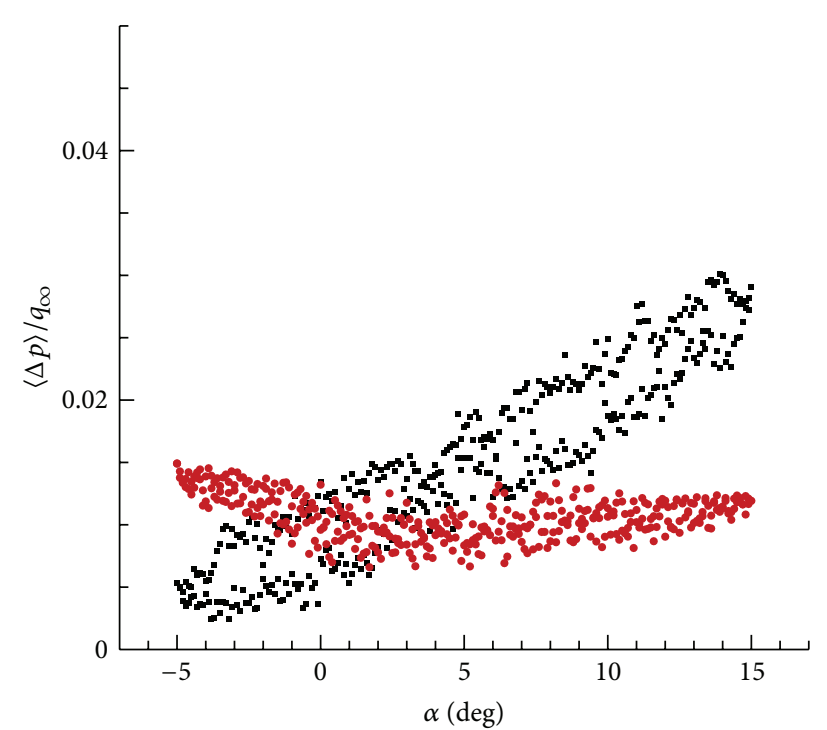

- WT ceiling - WT floor

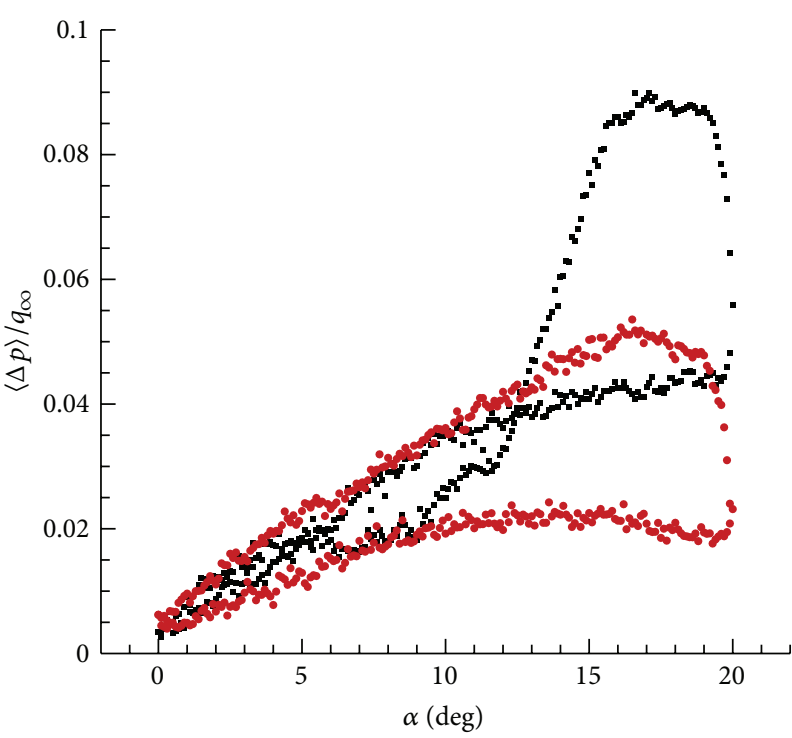

- WT ceiling - WT floor

(a) Light dynamic stall

(b) Deep dynamic stall

Figure 9: Pressure differences measured upstream and downstream the airfoil; pressure taps located, respectively, 3 chords upstream the airfoil leading edge and 2 chords downstream the airfoil trailing edge.

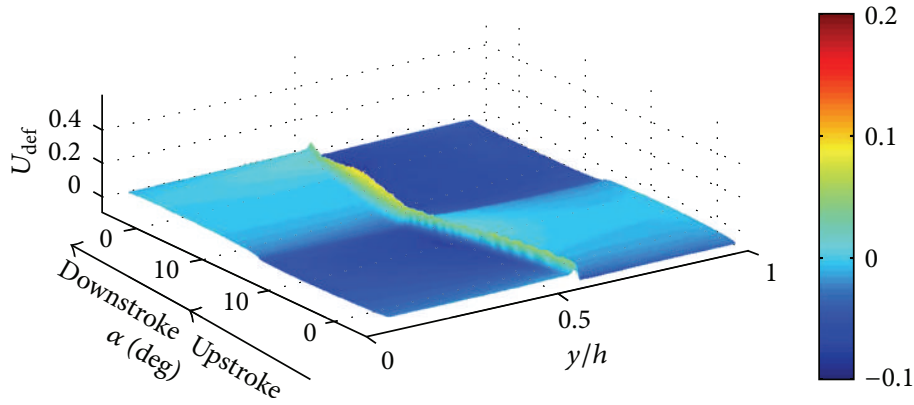

(a) Light dynamic stall

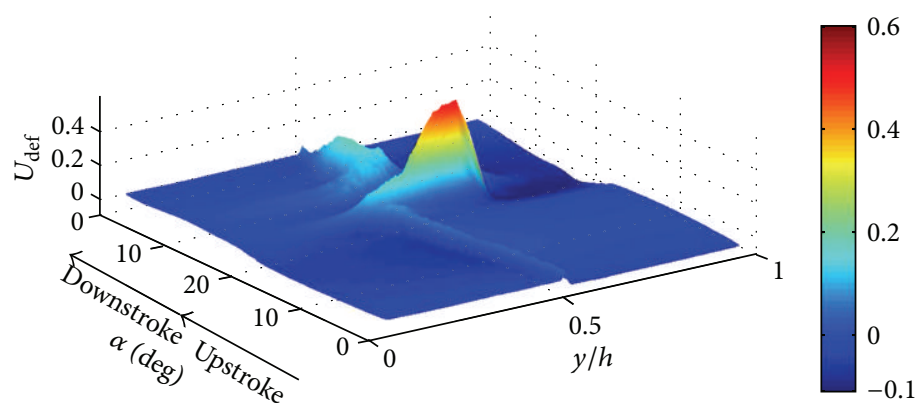

(b) Deep dynamic stall

FIGURE 10: Freestream adimensional velocity defect for the light dynamic stall (a) and the deep dynamic stall (b) conditions.

the wake due to the large vortical structures peculiar of this flow regime that are detached from the airfoil trailing edge (see Figure 10(b)). According to the occurrence of these flow phenomena, the turbulent kinetic energy in this phase of the motion is quite higher with respect to the light dynamic stall case, and in particular, its peak is about ten times greater (see Figure 11(b)).

In this phase of the pitching cycle, strong threedimensional secondary flows occur as it can be deduced from Figure 12 that shows the behavior of the adimensional bulk 


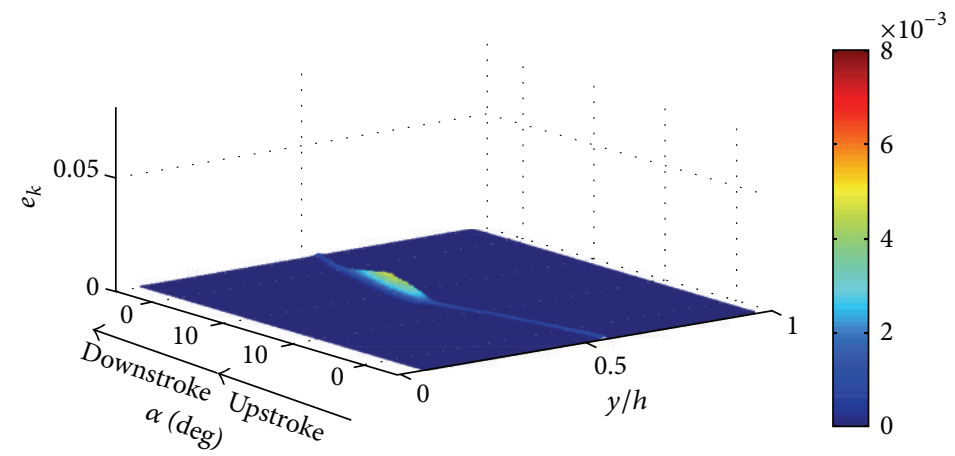

(a) Light dynamic stall

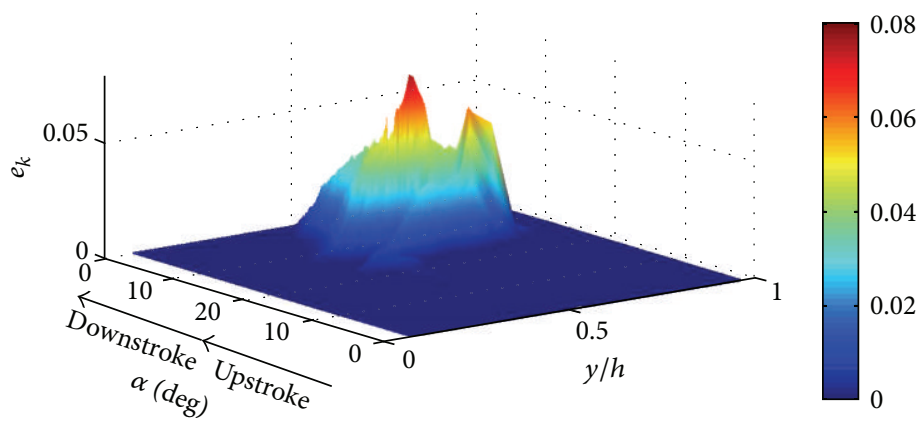

(b) Deep dynamic stall

FIGURE 11: Turbulent kinetic energy for the light dynamic stall (a) and the deep dynamic stall (b) conditions.

velocity for the light and the deep dynamic stall conditions. In particular, the bulk velocity defect observed in the deep dynamic stall curve from $12^{\circ}<\alpha<18^{\circ}$ in downstroke demonstrates that the flow is quite three dimensional in this range of angles of attack. The results of the threedimensional numerical simulations reported in Zanotti et al. [24, 25] carried out for the tested deep dynamic stall condition support this consideration. On the other hand, the quite flat behavior of the bulk velocity observed for the tested light dynamic stall condition suggests that in this regime three-dimensional flow effects are negligible during the whole pitching cycle.

\section{Conclusions}

An extensive wake survey was carried out downstream of a NACA 23012 pitching airfoil in light and deep dynamic stall conditions. The analysis of the wake velocity profiles highlights the predominant aerodynamic features involved in the different regimes of the dynamic stall and gives interesting information about the turbulent kinetic energy in the wake of a pitching airfoil. The wake survey analysis was supported by PIV measurements carried out on the airfoil upper surface, by the measurement of the lift and pitching moments and by the pressure measurements carried out on the wind tunnel floor and ceiling. Therefore, the experimental investigation gives a complete overview about the dynamic stall process over an oscillating airfoil.

The comprehensive experimental database can be considered a reference for the development and validation of

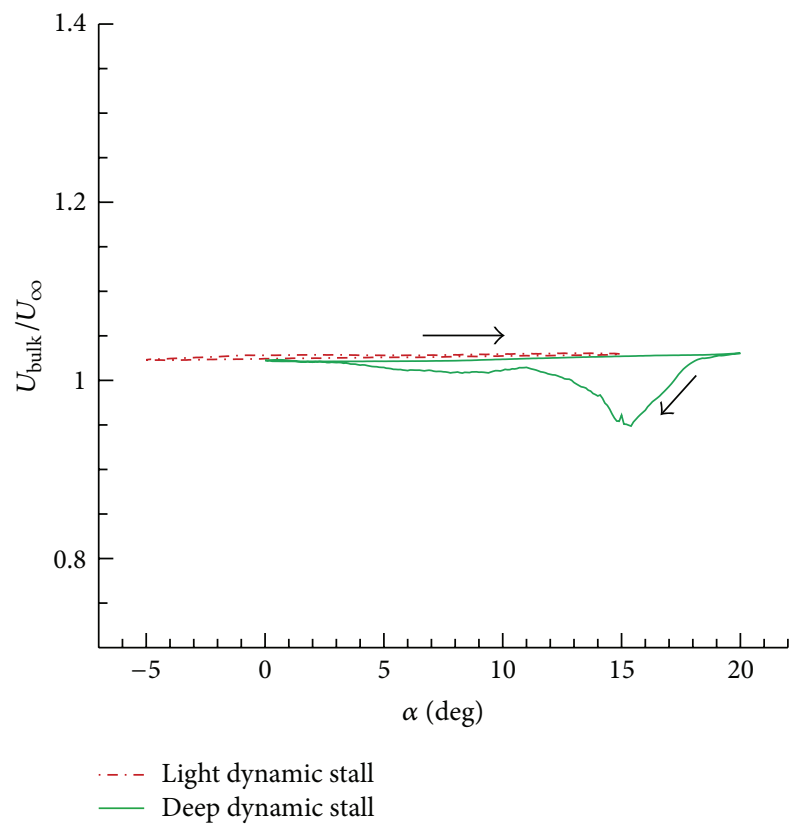

FIGURE 12: Wake bulk velocity for the light and deep dynamic stall conditions.

numerical tools. In particular, an interesting application would be the comparison of the wake velocity profiles with numerical simulations to evaluate the phase averaged total drag. Indeed, also on the side of the numerical simulation, 
the drag evaluation for a pitching airfoil in dynamic stall conditions is affected by a large uncertainty due to the severe unsteadiness that characterises this phenomenon. Thus, this problem is still quite open, and it represents a quite challenging activity.

\section{Nomenclature}

Symbols

$\begin{array}{ll}\alpha: & \text { Angle of attack } \\ \alpha_{m}: & \text { Mean angle of attack } \\ \alpha_{a}: & \text { Pitching oscillation amplitude } \\ \Delta p: & \text { Pressure difference measured upstream } \\ & \text { and downstream the airfoil } \\ \omega: & \text { Circular frequency, } \omega=2 \pi f \\ b: & \text { Blade section model span } \\ c: & \text { Blade section model chord } \\ C_{D}: & \text { Drag coefficient } \\ C_{L}: & \text { Lift coefficient } \\ C_{M}: & \text { Pitching moment coefficient about the } \\ & \text { airfoil quarter chord } \\ e_{k}: & \text { Adimensional turbulent kinetic energy } \\ f: & \text { Oscillation frequency } \\ h: & \text { Test section height } \\ k: & \text { Reduced frequency, } k=\pi f c / U_{\infty} \\ q_{\infty}: & \text { Freestream dynamic pressure } \\ \langle s\rangle: & \text { Phase-averaged signal } \\ U_{\infty}: & \text { Freestream velocity } \\ U_{\text {bulk }}: & \text { Wake bulk velocity } \\ U_{\text {def }}: & \text { Adimensional freestream velocity defect } \\ u: & \text { Streamwise velocity component } \\ v: & \text { Vertical velocity component } \\ u^{\prime}: & \text { Streamwise velocity component } \\ & \text { fluctuation } \\ v^{\prime}: & \text { Vertical velocity component fluctuation } \\ \langle u\rangle: & \text { Phase-averaged streamwise velocity } \\ x: & \text { component } \\ y: & \text { Streamwise coordinate } \\ z: & \text { Spanwise coordinate. } \\ & \\ h^{\prime} & \end{array}$

Abbreviations

DAER: Dipartimento di scienze e tecnologie aerospaziali

HW: Hot wire

PIV: Particle image velocimetry

Ma: Mach number

Re: Reynolds number.

\section{References}

[1] W. J. McCroskey, “The phenomenon of dynamic stall," NASA TM 81264, 1981.

[2] J. G. Leishman, Principles of Helicopter Aerodynamics, Cambridge University Press, 2006.
[3] F. O. Carta, "An analysis of the stall flutter instability of helicopter rotor blades," Journal of American Helicopter Society, vol. 12, pp. 1-8, 1967.

[4] A. D. Gardner, K. Richter, H. Mai, and D. Neuhaus, "Experimental control of compressible OA209 dynamic stall by air jets," in Proceedings of the 38th European Rotorcraft Forum, Amsterdam, The Netherlands, September 2012.

[5] M. S. Chandrasekhara, P. B. Martin, and C. Tung, "Compressible dynamic stall control using a variable droop leading edge airfoil," Journal of Aircraft, vol. 41, no. 4, pp. 862-869, 2004.

[6] M. L. Post and T. C. Corke, "Separation control using plasma actuators: dynamic stall vortex control on oscillating airfoil," AIA A Journal, vol. 44, no. 12, pp. 3125-3135, 2006.

[7] K. Mulleners and M. Raffel, "The onset of dynamic stall revisited," Experiments in Fluids, vol. 52, pp. 779-793, 2012.

[8] M. Raffel, J. Kompenhans, B. Stasicki, B. Bretthauer, and G. E. A. Meier, "Velocity measurement of compressible air flows utilizing a high-speed video camera," Experiments in Fluids, vol. 18, no. 3, pp. 204-206, 1995.

[9] A. Zanotti and G. Gibertini, "Experimental investigation of the dynamic stall phenomenon on a NACA 23012 oscillating aerofoil," Proceedings of the Institution of Mechanical Engineers, Part G: Journal of Aerospace Engineering, 2012.

[10] A. D. Gardner, K. Richter, H. Mai, A. R. M. Altmikus, A. Klein, and C. H. Rohardt, "Experimental Inves- tigation of dynamic stall performance for the EDI-M109 and EDI-M112 airfoils," in Proceedings of the 37th European Rotorcraft Forum, Gallarate, VA, Italy, September 2011.

[11] J. G. Leishman, "Dynamic stall experiments on the NACA 23012 aerofoil," Experiments in Fluids, vol. 9, no. 1-2, pp. 49-58, 1990.

[12] L. W. Carr, K. W. McAlister, and W. J. McCroskey, "Dynamic stall experiments on the NACA 0012 airfoil," NASA TP-1100, 1978.

[13] Y. W. Jung and S. O. Park, "Vortex-shedding characteristics in the wake of an oscillating airfoil at low Reynolds number," Journal of Fluids and Structures, vol. 20, no. 3, pp. 451-464, 2005.

[14] J. W. Chang, "Near-wake characteristics of an oscillating NACA 4412 airfoil," Journal of Aircraft, vol. 41, pp. 1240-1243, 2004.

[15] H. Sadeghi, M. Mani, and M. A. Ardakani, "Effect of amplitude and mean angle of attack on wake of an oscillating airfoil," Proceedings of World Academy of Science, Engineering and Technology, vol. 33, pp. 125-129, 2008.

[16] H. Sadeghi, M. Mani, and M. A. Ardakani, "Unsteady wake measurements behind an airfoil and prediction of dynamic stall from the wake," Aicraft Engineering and Aerospace Technology, vol. 82, pp. 225-236, 2010.

[17] T. Lee and P. Gerontakos, "Investigation of flow over an oscillating airfoil," Journal of Fluid Mechanics, vol. 512, pp. 313341, 2004.

[18] A. Zanotti, Retreating blade dynamic stall [Ph.D. thesis], Politecnico di Milano, 2012.

[19] A. Zanotti, F. Auteri, G. Campanardi, and G. Gibertini, "An experimental set up for the study of the retreating blade dynamic stall," in Proceedings of the 37th European Rotorcraft Forum, Gallarate, VA, Italy, September 2011.

[20] F. Durst, S. Noppenberger, M. Still, and H. Venzke, "Influence of humidity on hot-wire measurements," Measurement Science and Technology, vol. 7, no. 10, pp. 1517-1528, 1996.

[21] P. Wernert and D. Favier, "Considerations about the phase averaging method with application to ELDV and PIV measurements over pitching airfoils," Experiments in Fluids, vol. 27, no. 6, pp. 473-483, 1999. 
[22] T. Nishino and K. Shariff, "Numerical study of wind-tunnel sidewall effects on circulation control airfoil flows," AIAA Journal, vol. 48, no. 9, pp. 2123-2132, 2010.

[23] G. K. Batchelor, An Introduction to Fluid Dynamics, Cambridge University Press, 2000.

[24] A. Zanotti, S. Melone, R. Nilifard, and A. D’Andrea, "Experimental-numerical investigation of a pitching airfoil in deep dynamic stall," Proceedings of the Institution of Mechanical Engineers, Part G: Journal of Aerospace Engineering, 2013.

[25] A. Zanotti, R. Nilifard, G. Gibertini, A. Guardone, and G. Quaranta, "Experimental-numerical investigation of the dynamic stall phenomenon over the NACA 23012 airfoil," in Proceedings of the 38th European Rotorcraft Forum, Amsterdam, The Netherlands, September 2012. 

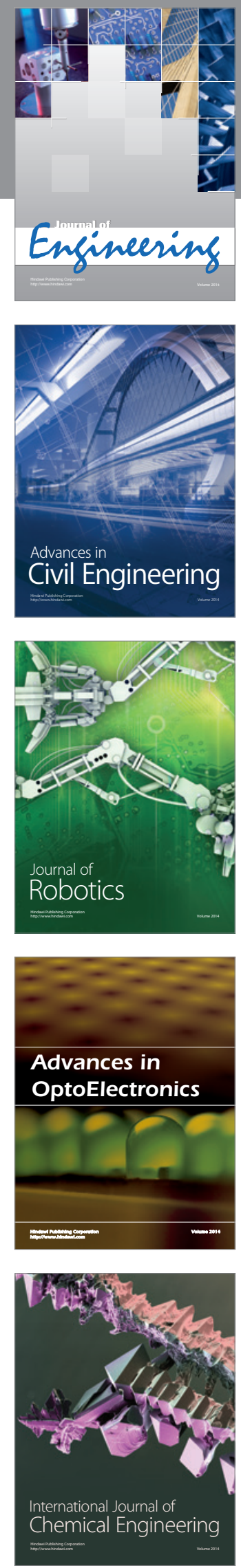

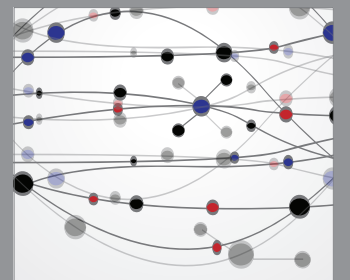

The Scientific World Journal
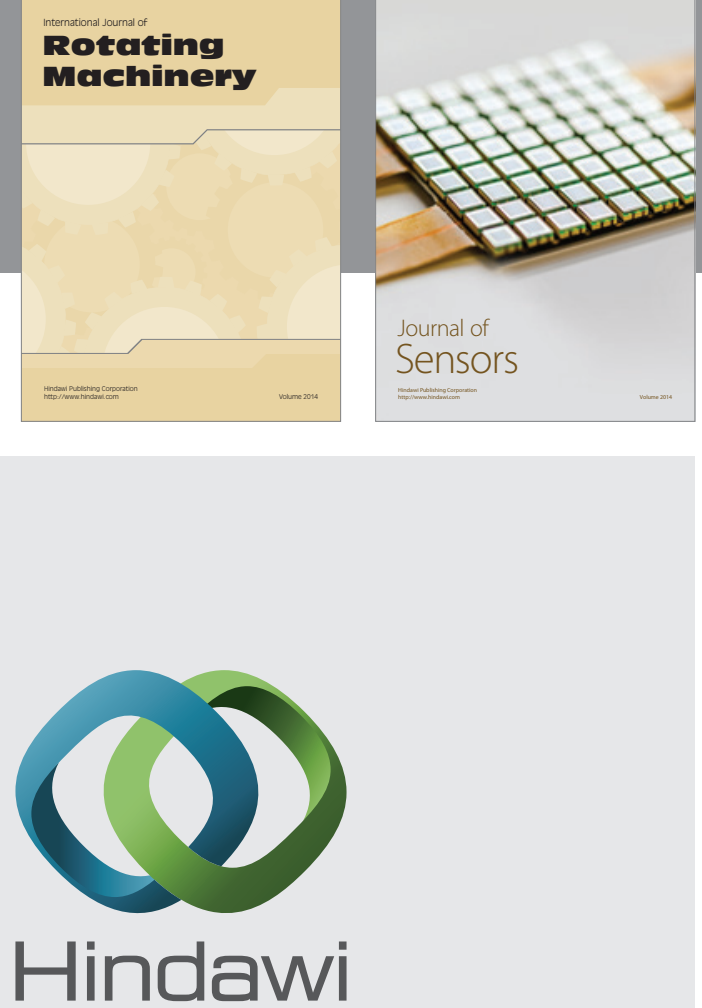

Submit your manuscripts at http://www.hindawi.com
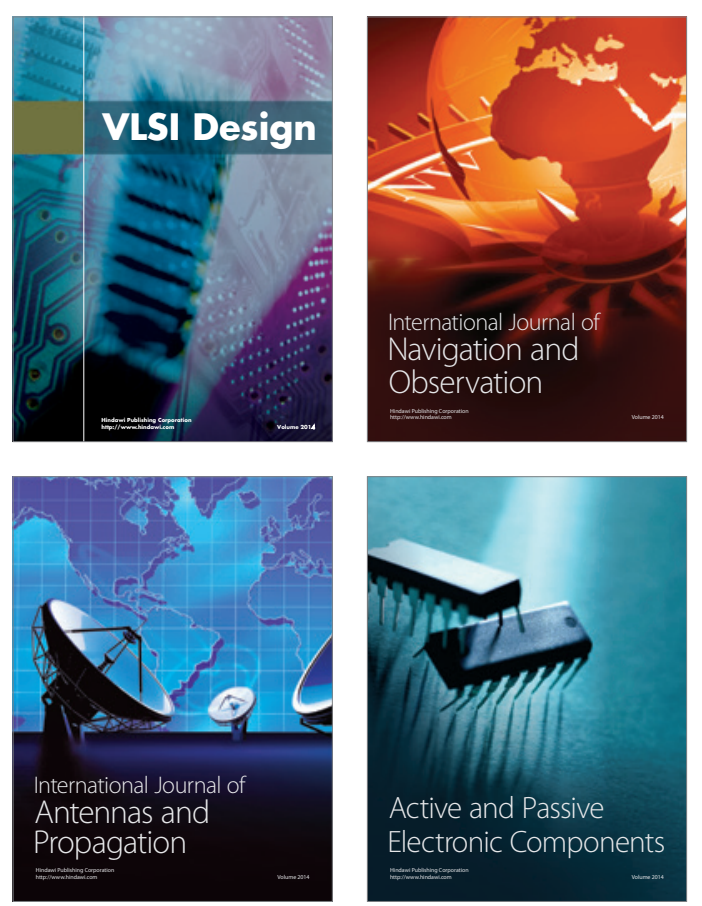
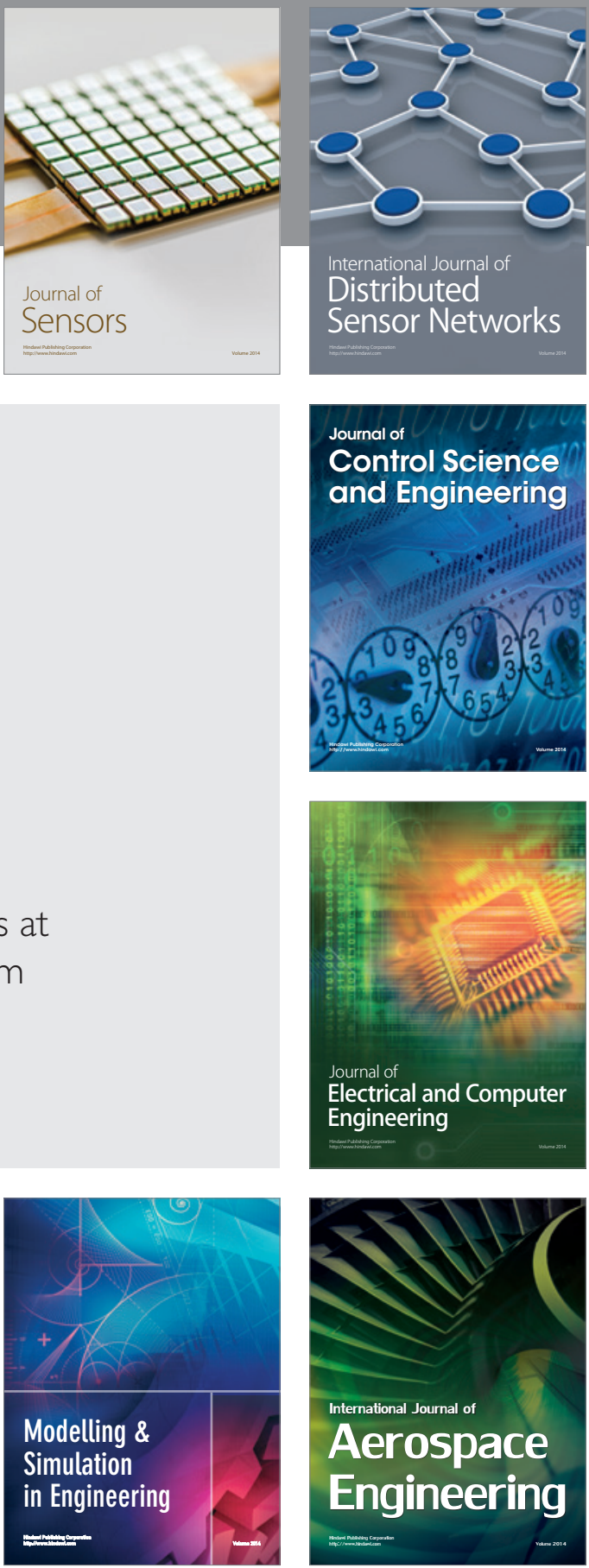

Journal of

Control Science

and Engineering
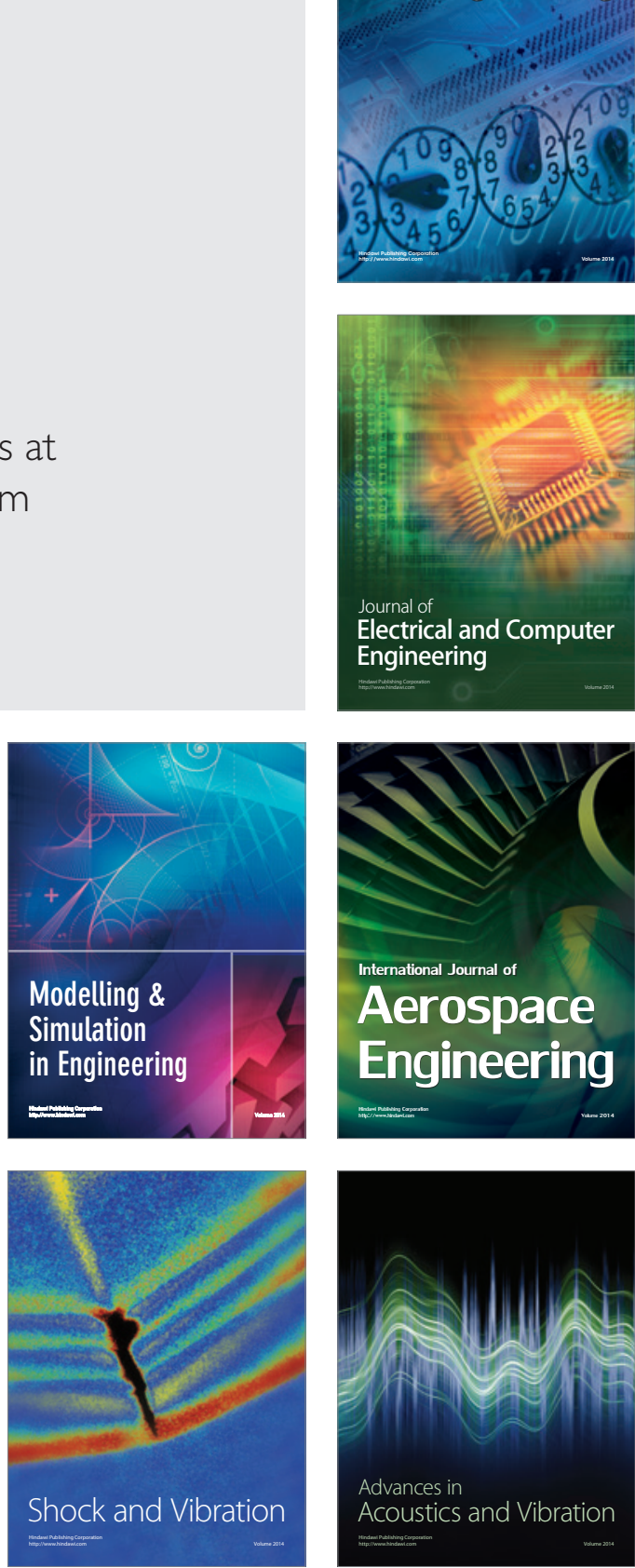